\title{
Capacidade funcional e qualidade de vida de pacientes com feridas crônicas
}

\section{Functional capacity and quality of life of patients with chronic wounds}

\section{Capacidade funcional e qualidade de vida de pacientes com feridas crônicas}

Aline Costa de Oliveira, Andressa dos Santos Alvarenga, Franciane de Sousa Freitas, Daniel de Macêdo Rocha, Sandra Marina Gonçalves Bezerra, Lídya Tolstenko Nogueira

ORCID IDs

Oliveira AC (iD https://orcid.org/0000-0003-1738-4808

Alvarenga AS (iD https://orcid.org/0000-0001-7343-0560

Freitas FS (iD https://orcid.org/0000-0002-9595-6659

Rocha DM (iD https://orcid.org/0000-0003-1709-2143

Bezerra SMG (D) https://orcid.org/0000-0003-3890-5887

Nogueira LT (D) https://orcid.org/0000-0003-4918-6531

\section{COMO CITAR}

Oliveira AC, Alvarenga AS, Freitas FS, Rocha DM, Bezerra SMG, Nogueira LT. Capacidade funcional e qualidade de vida de pacientes com feridas crônicas. ESTIMA, Braz. J. Enterostomal Ther., 16: e2918. https://doi.org/10.30886/estima.v16.612_PT

\section{RESUMO}

Objetivo: Avaliar a capacidade funcional e a qualidade de vida (QV) de pacientes com feridas crônicas. Métodos: Pesquisa de caráter transversal analítico composta por 74 pacientes com feridas crônicas acompanhadas em um centro de referência para tratamento de feridas complexas em Teresina, estado do Piauí. Para coleta de dados, utilizaram-se os instrumentos índice de Katz e Cardiff Wound Impact Schedule. Resultados: Dentre os domínios, o "bem-estar" apresentou menor escore médio e o grau de dependência obteve associação significativa com os domínios bem-estar e "vida social". As atividades banho, vestir-se, higiene pessoal e locomoção apresentaram associação entre os domínios de QV. Conclusão: O grau de independência em realizar as atividades básicas de vida diária, com destaque para o banho, o vestir-se e a higiene pessoal, apresentaram maiores escores entre os domínios, indicando melhor QV. Espera-se que a implantação de medidas que auxiliem a preservar a capacidade funcional proporcione melhoria na QV dos pacientes.

DESCRITORES: Ferimentos e lesões; Qualidade de vida; Atividades cotidianas; Estomaterapia. 


\section{ABSTRACT}

Objective: To evaluate functional capacity and quality of life (QoL) of patients with chronic wounds. Methods: A cross-sectional analytical study composed of 74 patients with chronic wounds accompanied at a reference center for the treatment of complex wounds in Teresina, state of Piaui. Data were collected using the Katz index and the Cardiff Wound Impact Schedule. Results: Among the domains, the "well-being" had a lower mean score and the degree of dependence obtained a significant association with the well-being and "social life" domains. The bathing, dressing, personal hygiene and locomotion activities showed an association between the domains of QoL. Conclusion: The degree of independence in performing the basic activities of daily living, especially bathing, dressing and personal hygiene, presented higher scores between domains, indicating a better QoL. It is expected that the implantation of measures that help to preserve the functional capacity will improve the patients' QoL.

DESCRIPTORS: Wounds and injuries; Quality of life; Daily activities; Stomatherapy.

\section{RESUMEN}

Objetivo: Evaluar la capacidad funcional y calidad de vida (CV) de pacientes con heridas crónicas. Métodos: Estudio de carácter transversal analítico compuesto por 74 pacientes con heridas crónicas con seguimiento en un centro de referencia para tratamiento de heridas complejas en Teresina, Estado de Piauí. Para la recolección de datos, se utilizaron los instrumentos índices de Katz y Cardiff Wound Impact Schedule. Resultados: Entre los dominios, el «bienestar» presentó menor puntuación promedio y el grado de dependencia obtuvo asociación significativa con los dominios bienestar y «vida social» Las actividades del baño, vestirse, higiene personal y movilidad presentaron asociación entre los dominios de CV. Conclusión: El grado de independencia para realizar las actividades básicas de vida diaria, destacando las del baño, vestirse y la higiene personal, presentaron mayores puntuaciones entre los dominios, indicando mejor CV. Se espera que la implementación de medidas que ayuden a preservar la capacidad funcional proporcione mejora en la CV de los pacientes.

DESCRIPTORES: Heridas y lesiones; Calidad de Vida; Actividades cotidianas; Estomaterapia.

\section{INTRODUÇÃO}

As feridas crônicas referem-se a lesões cutâneas com retardo no processo de cicatrização, compreendendo um período superior a 6 semanas e que apresentam alto índice de recorrências. Essas feridas são caracterizadas por um atraso no reparo fisiológico que leva a um estado inflamatório patológico e provoca alterações físicas, psicossociais e econômicas nas pessoas por elas acometidas ${ }^{1,2}$.

A cronificação das feridas tem se tornado uma preocupação tanto para os profissionais da área quanto para os sistemas de saúde, pois está associada a tratamentos contínuos, dispendiosos e prolongados, com consequências inevitáveis nas diferentes dimensões da vida do doente/ família e, consequentemente, na sua qualidade de vida (QV) e capacidade funcional ${ }^{3}$.

As feridas crônicas podem ocasionar dor, edema e prejudicar a mobilidade, fatores que repercutem negativamente sobre a capacidade funcional dos pacientes, tendo em vista que acarretam problemas relacionados ao convívio social, perda de autoestima, autocuidado precário, e, consequentemente, comprometem a $\mathrm{QV}^{4}$.

A QV compreende "a percepção do indivíduo sobre a sua posição na vida, no contexto da cultura e dos sistemas de valores nos quais ele vive, e em relação a seus objetivos, expectativas, padrões e preocupações”. . A avaliação da QV é considerada uma medida de desfecho em saúde, pois atende ao pressuposto de individualizar a atenção e de considerar a subjetividade da pessoa atendida ${ }^{6}$, tornando-se nítida a necessidade de realização de estudos que abranjam essa temática e as alternativas terapêuticas existentes na atualidade ${ }^{7}$.

Segundo a classificação internacional de comprometimento, incapacidades e desvantagens da World Health Organization (WHO), a capacidade funcional é considerada a ausência de dificuldades para se executar as atividades cotidianas ${ }^{8}$. A capacidade funcional é fundamental para manutenção do bem-estar e da autonomia das pessoas e a presença das feridas pode 
desenvolver ou influenciar uma situação de dependência, afetando negativamente as dimensões físicas, sociais e psicológicas relacionadas diretamente à QV. Assim, a avaliação da capacidade funcional permite investigar a independência para execução das atividades básicas e se torna relevante na determinação das condições de saúde das pessoas e no desenvolvimento de intervenções para melhoria na vida dessas?.

Ressalta-se que ainda são escassos os estudos que abordam a $\mathrm{QV}$ e a capacidade funcional em pessoas com feridas crônicas, no entanto, a compreensão das condições vividas por essa população é fundamental aos profissionais de saúde, visto que o processo de reparação lento das feridas crônicas provoca perdas na autoestima e condições de incapacidades ${ }^{4}$. Assim, reafirma-se a necessidade do apoio profissional, principalmente em mudanças de condutas baseadas nas reais necessidades dos pacientes, priorizando a redução de complicações, além de auxiliar nos processos de aceitação e adaptação.

Diante disso, o objetivo deste estudo foi avaliar a capacidade funcional e $\mathrm{QV}$ de pacientes com feridas crônicas.

\section{MÉTODOS}

Estudo descritivo, transversal, analítico, com abordagem quantitativa, desenvolvido em um centro de referência para tratamento de feridas complexas de Teresina, estado do Piaú, Brasil, no período de fevereiro a maio de 2017. A população foi constituída de pacientes atendidos no ambulatório de feridas complexas para avaliação e tratamento da lesão no período da coleta de dados, totalizando 74 pacientes. Como critérios de inclusão, foram consideradas pessoas com idade igual ou superior a 18 anos e com tempo de existência da lesão superior a 6 semanas, e os de exclusão focaram-se em pessoas com comprometimento cognitivo para preenchimento dos questionários e em idosos que não atingiram a pontuação mínima na avaliação mental.

A coleta de dados foi norteada por um formulário adaptado para caracterização sociodemográfica (sexo, idade, procedência, estado civil, renda e escolaridade) e clínica (fatores de risco, etiologia, localização anatômica, tempo de existência, tipo de tecido, quantidade e aspecto do exsudato, extensão inicial e final, coberturas e produtos utilizados e frequência entre as trocas ${ }^{10} \mathrm{e}$ pelo Questionário de Avaliação Mental ${ }^{11}$ aplicado em pessoas com idade igual ou maior que 60 anos, sendo selecionadas as que obtiveram no mínimo sete acertos.
Ainda, utilizou-se o questionário CardiffWound Impact Schedule (CWIS) para mensuração da $\mathrm{QV}^{12}$ e o índice de $\mathrm{Katz}^{13}$ para avaliação da capacidade funcional. O CWIS consiste em uma ferramenta específica e autoaplicável, validado na versão brasileira ${ }^{14}$, com boa consistência interna (Cronbach $\alpha=0,920$ ) e composto por 47 itens distribuídos nos domínios "bem-estar" (sete itens), "sintomas físicos e vida diária"(24 itens), "vida social" (14 itens) e autoavaliação da $\mathrm{QV}^{2}$. Os itens são classificados em escala do tipo Likert e transformados em escores que variam de 0 a 100, em que quanto maior a pontuação, melhor será a $\mathrm{QV}^{12}$. Já o índice de Katz é comumente empregado para mensurar o grau de independência e a capacidade funcional no desempenho de seis funções de vida diária, sendo elas: alimentação, continência, transferência (locomoção), ir ao banheiro, vestir-se e tomar banho ${ }^{13,15}$.

Os dados foram digitados em planilhas de dupla entrada no Microsoft Office Excel e posteriormente exportados para o programa Statistic Package for the Social Sciences (SPSS) versão 22, visando a análise descritiva e inferencial. Foram calculadas as medidas de tendências centrais (média e mediana), de dispersão (desvio-padrão) e valores mínimos e máximos para as variáveis quantitativas e proporções para as categóricas. $\mathrm{Na}$ análise inferencial, foram realizados o teste de KolmogorovSmirnov para verificação da normalidade dos dados, o teste de Kruskal Wallis e $U$ de Mann-Whitney (testes comparativos não paramétricos), o teste $t$ de Student e analysis of variance (ANOVA) (testes comparativos paramétricos). $\mathrm{O}$ critério de determinação de significância adotado no estudo foi o nível de $5 \%(\mathrm{p}<0,05)$.

Os participantes que aceitaram fazer parte do estudo assinaram o Termo de Consentimento Livre Esclarecido (TCLE). Esta pesquisa seguiu os preceitos éticos e legais contidos na Resolução 466/2012 do Conselho Nacional de Saúde e foi aprovada pelo Comitê de Ética em Pesquisa da Universidade Federal do Piaú sob parecer 1.837.210.

\section{RESULTADOS}

Dos 74 participantes, 39 (52,7\%) eram do sexo masculino, 31 (41,9\%) situados na faixa etária entre $41-59$ anos e 32 aposentados $(43,2 \%)$. Quanto à mobilidade, 35 (47,3\%) deambulavam sem auxilio. Houve predominância de feridas de origem vasculogênica em $26(35,1 \%)$ pessoas e traumáticas em 22 (29,7\%).

Sobre a QV, a Tabela 1 apresenta os escores do questionário CWIS. O domínio com menor escore médio foi o bem-estar, 
apresentando média de 33,2 17 ,2, enquanto os domínios

Tabela 1. Escores dos domínios de qualidade de vida (QV) dos pacientes com feridas crônicas, Teresina, estado do Piauí, Brasil, $2018(n=74)$.

\begin{tabular}{lccc} 
& \multicolumn{3}{c}{ Escores } \\
\cline { 2 - 4 } Domínios & $\begin{array}{c}\text { Média } \pm \\
\text { desvio-padrão }\end{array}$ & Mínimo & Máximo \\
\hline Bem-estar & $33,2 \pm 17,2$ & 0,0 & 85,7 \\
\hline Sintomas físicos e & $66,9 \pm 17,5$ & 27,1 & 94,8 \\
vida diária & $65,6 \pm 19,7$ & 17,9 & 100,0 \\
\hline Vida social & $7,2 \pm 2,4$ & 1,0 & 10,0 \\
\hline Q & $7,6 \pm 2,1$ & 1,0 & 10,0 \\
\hline
\end{tabular}

sintomas físicos e vida diária e vida social apresentaram

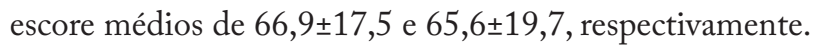

Com relação à capacidade funcional, verificou-se que $66(89,2 \%)$ pacientes realizavam suas atividades básicas de vida diária. Quanto à associação entre grau de dependência e $\mathrm{QV}$, os domínios bem-estar e vida social foram significativos e as pessoas independentes diferiam estatisticamente das dependentes no domínio vida social (Tabela 2).

A avaliação da execução das atividades banho, vestir-se, higiene pessoal, locomoção, continência e alimentação evidenciou maior frequência de pacientes independentes em todas as atividades (Tabela 3 ).

Tabela 2. Associação entre o grau de dependência e a qualidade de vida dos pacientes com feridas crônicas, Teresina, estado do Piauí, Brasil, $2018(n=74)$.

\begin{tabular}{|c|c|c|c|c|c|c|c|}
\hline \multicolumn{8}{|c|}{ Domínios do Cardiff Wound Impact Schedule (CWIS) } \\
\hline $\begin{array}{c}\text { Grau de } \\
\text { dependência }\end{array}$ & n (\%) & Bem-estar ${ }^{*}$ & $\mathrm{p}^{\dagger}$ & $\begin{array}{c}\text { Sintomas físicos e } \\
\text { vida diária* }\end{array}$ & $\mathrm{p}^{\dagger}$ & Vida socialł & $p^{\S}$ \\
\hline Independente & $66(89,2)$ & $32,1(0,0-85,7)$ & & $71,9(27,1-94,8)$ & & $67,3 \pm 18,5$ & \\
\hline Intermediário & $2(2,7)$ & $48,2(39,3-57,1)$ & 0,043 & $69,79(65,6-74,0)$ & 0,087 & $72,3 \pm 3,8$ & 0,017 \\
\hline Dependente & $6(8,7)$ & $17,9(0,0-39,3)$ & & $48,9(39,6-75,0)$ & & $44,0 \pm 20,0$ & \\
\hline
\end{tabular}

* Mediana (mínimo-máximo); † p-valor de Kruskall Wallis; ${ }^{\ddagger}$ Média \pm desvio-padrão; ${ }^{\S}$ Analysis of variance (ANOVA).

Tabela 3. Associação entre as atividades básicas de vida diária e os domínios de qualidade de vida, Teresina, estado do Piauí, Brasil, $2018(n=74)$.

\begin{tabular}{|c|c|c|c|c|c|c|c|}
\hline \multirow{2}{*}{$\begin{array}{l}\text { Atividades básicas } \\
\text { de vida diária }\end{array}$} & \multirow[b]{2}{*}{$\mathrm{n}(\%)$} & \multicolumn{6}{|c|}{ Domínios do Cardiff Wound Impact Schedule (CWIS) } \\
\hline & & Bem-estar ${ }^{*}$ & $\mathbf{p}^{\dagger}$ & $\begin{array}{c}\text { Sintomas físicos e } \\
\text { vida diária* }\end{array}$ & $p^{\dagger}$ & Vida social $^{*}$ & $\mathbf{p}^{\dagger}$ \\
\hline \multicolumn{8}{|l|}{ Banho } \\
\hline Independente & $69(93,2)$ & $32,1(0,0-85,7)$ & 0,006 & $72,9(27,1-94,8)$ & 0,007 & $69,6(23,2-100,0)$ & 0,009 \\
\hline Dependente & $5(6,8)$ & $14,3(0,0-28,6)$ & & $46,9(39,6-54,2)$ & & $50,0(17,9-51,8)$ & \\
\hline \multicolumn{8}{|l|}{ Vestir-se } \\
\hline Independente & $70(94,6)$ & $32,1(0,0-85,7)$ & 0,018 & $71,9(27,1-94,8)$ & 0,025 & $69,6(23,2-100,0)$ & 0,030 \\
\hline Dependente & $4(5,4)$ & $12,5(0,0-28,6)$ & & $48,9(39,6-54,2)$ & & $50,0(17,9-51,8)$ & \\
\hline \multicolumn{8}{|l|}{ Higiene pessoal } \\
\hline Independente & $67(90,5)$ & $32,1(0,0-85,7)$ & 0,165 & $68,2 \pm 17,4 \S$ & $0,056 \neq$ & $67,3 \pm 18,7 \S$ & $0,015 \neq$ \\
\hline Dependente & $7(9,5)$ & $17,9(0,0-57,1)$ & & $54,9 \pm 14,2 \S$ & & $48,5 \pm 21,7 \S$ & \\
\hline \multicolumn{8}{|l|}{ Locomoção } \\
\hline Independente & $67(90,5)$ & $31,1(0,0-85,7)$ & 0,099 & $72,9(27,1-94,8)$ & 0,034 & $69,6(25,0-67,4)$ & 0,024 \\
\hline Dependente & $7(9,5)$ & $17,9(0,0-39,3)$ & & $51,0(39,6-75,0)$ & & $50,0(17,9-47,7)$ & \\
\hline \multicolumn{8}{|l|}{ Continência } \\
\hline Independente & $71(95,9)$ & $32,1(0,0-85,7)$ & 0,086 & $69,8(27,1-94,8)$ & 0,366 & $69,4(17,9-100,0)$ & 0,388 \\
\hline Dependente & $3(4,1)$ & $17,9(7,1-28,6)$ & & $54,2(46,9-75,0)$ & & $51,8(50,0-71,4)$ & \\
\hline \multicolumn{8}{|l|}{ Alimentação } \\
\hline Independente & $72(97,3)$ & $32,1(0,0-85,7)$ & 0,170 & $70,3(27,1-94,8)$ & 0,172 & $69,6(17,9-100,0)$ & 0,243 \\
\hline Dependente & $2(2,7)$ & $17,9(7,1-28,6)$ & & $50,5(46,9-54,2)$ & & $50,9(50,0-51,8)$ & \\
\hline
\end{tabular}

* Mediana (mínimo-máximo); ${ }^{\dagger}$ Teste $U$ de Mann Whitney; ${ }^{\ddagger}$ Teste $t$ de Student; ${ }^{\S}$ Média \pm desvio-padrão. 
Com relação às atividades básicas de vida diária e à $\mathrm{QV}$, o banho apresentou associação significativa com todos os domínios bem-estar $(\mathrm{p}=0,006)$, sintomas físicos e vida diária $(p=0,007)$ e vida social $(p=0,009)$. O ato de vestir-se apresentou associação com os domínios bem-estar $(\mathrm{p}=0,018)$, sintomas físicos e vida diária $(p=0,025)$ e vida social $(p=0,030)$. A higiene pessoal foi positivamente associada ao domínio vida social $(p=0,015)$ e a locomoção foi significativa quando associada aos domínios sintomas físicos e vida diária $(\mathrm{p}=0,034)$ e vida social $(\mathrm{p}=0,024)$.

\section{DISCUSSÃO}

A caracterização das variáveis sociodemográficas identificou que a maioria dos pacientes era do sexo masculino e situados na faixa etária entre 41-59 anos, gerando repercussões diretas nos serviços de saúde e no ambiente domiciliar, uma vez que a cronicidade das feridas promove o aumento de custos e leva ao afastamento das atividades laborais ou a aposentadoria precoce ${ }^{5}$, sendo essa a situação ocupacional predominante nos participantes do estudo.

Cerca de $47,3 \%$ da população estudada deambulava sem auxilio e apresentava feridas de origem vasculogênica. Essas lesões geralmente são acompanhadas por dor intensa, exsudado e odor, apresentando cicatrização lenta que, somada aos fatores psicossociais e à dependência de cuidados, repercute sobre a capacidade funcional e afeta a $\mathrm{QV}^{16,17}$.

O domínio bem-estar apresentou escore médio menor de $\mathrm{QV}$, enquanto os domínios sintomas físicos e vida diária e vida social apresentaram escores médios semelhantes e maiores. Esses resultados são compatíveis com outros estudos que também utilizaram o questionário CWIS para avaliar a QV de pessoas com feridas ${ }^{18,19}$.

O bem-estar está relacionado aos níveis de ansiedade, envolvendo a percepção emocional que se relaciona com a existência de emoções positivas e ausência de emoções negativas, envolvendo fatores como satisfação de vida, habilidades de enfrentamento, otimismo e perspectiva para o futuro ${ }^{19,20}$. Pacientes com úlceras frequentemente experimentam emoções negativas como vergonha, constrangimento, depressão, preocupação de que a condição piore, apatia e problemas mentais, coexistindo com forte sentimento de solidão ${ }^{6}$.
Outros estudos também revelaram impacto negativo de lesões de pele na $\mathrm{QV}$ relacionada à esfera emocional, principalmente em pacientes com úlceras crônicas ${ }^{6,21}$. O caráter progressivo e crônico das feridas afeta as esferas mais importantes da vida humana, sendo importante a presença de cuidados abrangentes que cubram todos os aspectos do funcionamento prejudicado nas esferas física, psicossocial e emocional ${ }^{22}$.

No presente estudo, avaliou-se, ainda, a capacidade funcional, identificando baixo impacto referente ao grau de dependência, pois $89,2 \%$ das pessoas apresentavam-se independentes para todas as atividades ${ }^{23}$. No entanto, quando associados à $\mathrm{QV}$, os domínios bem-estar e vida social foram significativos.

Estudos anteriores mostraram que as incapacidades a longo prazo, particularmente aquelas que afetam as atividades diárias de rotina, têm correlação indireta com a saúde mental e com a $\mathrm{QV}^{24,25}$. Depender de outras pessoas para o desempenho de assuntos cotidianos é resultado previsível perante a $\mathrm{QV}$ comprometida. Assim, o fornecimento de serviços adequados a essas pessoas pode reduzir sua dependência e ter impacto positivo no aumento de sua $\mathrm{QV}^{25}$.

Os resultados revelam que a maioria dos participantes não apresentou dificuldade no banho, para vestir-se, realizar sua higiene pessoal, na locomoção, continência e alimentação. Essas atividades diárias não interferem diretamente na vida dos pacientes com feridas crônicas, vez que para executar essas atividades utilizam-se, também, os membros superiores, região do corpo menos afetada por feridas ${ }^{26}$.

No caso das atividades diárias, o banho apresentou associação em todos os domínios bem-estar, sintomas físicos e vida diária e vida social. $\mathrm{O}$ ato de vestir-se apresentou associação com os domínios bem-estar, sintomas físicos e vida diária e vida social. A higiene pessoal foi positivamente associada ao domínio vida social e a locomoção foi significativa quando associada aos domínios sintomas físicos e vida diária e vida social. Destaca-se que é evidenciada, na literatura, a relação entre a presença de feridas e o comprometimento do bem-estar, uma vez que é comum a ocorrência de limitações físicas e de dificuldades no desenvolvimento de atividades diárias, sociais e laborais, bem como a dependência de cuidados ${ }^{27}$.

A presença de comprometimentos no autocuidado foi evidenciada por dificuldades para deambulação, em vestir-se e no uso do banheiro, também verificada em outros estudos que avaliaram os impactos da lesão na capacidade funcional 
dos pacientes ${ }^{28}$. Assim, considera-se que esse problema pode refletir diretamente no cuidado à sua própria ferida, presumindo a dependência de cuidados e a necessidade contínua de apoio dos familiares e dos profissionais de saúde.

Segundo a literatura, o motivo das limitações das atividades diárias em pacientes com feridas é influenciando também pelos curativos, recidivas e elevado tempo de tratamento que podem causar dificuldades ao movimentar-se ou pela necessidade de prevenção de complicações ${ }^{29}$. Além disso, essas limitações podem estar associadas à presença de intensa dor e exsudação e cheiro desagradável da ferida ${ }^{6}$, influenciando, dessa maneira, em níveis mais baixo de $\mathrm{QV}$ e comprometimentos da capacidade funcional.

\section{CONCLUSÃO}

Este estudo demonstrou que a presença de feridas crônicas compromete negativamente a $\mathrm{QV}$ dos pacientes e que o domínio bem-estar interferiu diretamente para pior $\mathrm{QV}$, gerando impactos físicos, sociais e emocionais. Além disso, observou-se significância entre o grau de independência em realizar as atividades básicas de vida diária, com destaque para o banho, o vestir-se, a higiene pessoal e os maiores escores entre os domínios, o que gerou melhoria na $\mathrm{QV}$.

Assim, a implantação de medidas que auxiliem a preservar a capacidade funcional e que proporcionem a realização das atividades diárias e do autocuidado implica na possibilidade de uma vida autônoma e socialmente ativa. Desse modo, tornam-se necessárias pesquisas futuras com foco nas intervenções abrangentes que promovam a reabilitação das pessoas com feridas na execução de suas atividades e a melhoria da QV.

\section{CONTRIBUIÇÃO DOS AUTORES}

Conceitualização, de Oliveira AC e Rocha DM; Metodologia, de Oliveira AC e Rocha DM; Investigação, Alvarenga AS e Freitas FS; Redação - Primeira versão, de Oliveira AC; Alvarenga AS e Freitas FS; Redação - Revisão \& Edição, Nogueira LT e Bezerra SMG; Aquisição de Financiamento, de Oliveira AC; Alvarenga AS e Freitas FS; Recursos, Bezerra SMG; Supervisão, Nogueira LT.

\section{REFERÊNCIAS}

1. Markova A, Mostow EN. US skin disease assessment: ulcer and wound care. Dermatol Clin. 2012;30(1):107-11. https:// doi.org/10.1016/j.det.2011.08.005.

2. Shubhangi VA. Chronic leg ulcers: epidemiology, aetiopathogenesis and management. Ulcers. 2013;2013:1-9. https://doi.org/10.1155/2013/413604.

3. Mestre T, Rodrigues A, Cardoso J. Cicatrização de feridas crônicas: algumas opções terapêuticas. Rev SPDV. 2012;70(4):423-31. https://doi.org/10.29021/spdv.70.4.96.

4. Joaquim FL, Camacho ACLF, Sabóia VM, Santos RC, Santos LS, Nogueira GA. Impacto da visita domiciliar na capacidade funcional de pacientes com úlceras venosas. Rev Bras Enferm. 2016;69(3):468-77. https://doi.org/10.1590/00347167.2016690308i.

5. The WHOQOL Group. World Health Organization quality of life assessment (WHOQOL): position paper from World Health Organization. Soc Sci Med. 1995;41(10):1403-9. https://doi.org/10.1016/0277-9536(95)00112-K.

6. Szewczyk MT, Moscicka P, Jawien A, BialaskJC, Cierzniakowsk K, Slusarz R, et al. Quality of life in patients with leg ulcers or skin lesions-A pilot study. Postępy Dermatol Allergol. 2015;32(6):465-9. https://doi.org/10.5114/pdia.2014.40983.

7. Paula GR, Souza BN, Santos LF, Barbosa MA, Brasil W, Oliveira LMAC. Qualidade de vida para a avaliação de grupos de promoção da saúde. Revista Bras Enferm. 2016;69(2):242-9. https://doi.org/10.1590/0034-7167.2016690206i.
8. Alves LC, Leite IC, Machado CJ. Conceituando e mensurando a incapacidade funcional da população idosa: uma revisão de literatura. Ciênc saúde coletiva. 2008;13(4):1199-207. https://doi.org/10.1590/S1413-81232008000400016.

9. Joaquim FL, Camacho ACLF, Silva RMCRA, Leite BS, Queiroz RS, Assis CRC. Impact of home visits on the functional capacity of patients with venous ulcers. Rev Bras Enferm. 2017;70(2):287-93.https://doi.org/10.1590/0034-7167.2016 $\underline{690308 i}$.

10. Bezerra SMG, Rocha DM, Nogueira LT. Protocolo de prevenção, avaliação e tratamento de lesões pele do serviço público municipal de Teresina. Teresina: Prefeitura Municipal de Teresina; 2016.

11. Ventura MM, Bottino CMC. Avaliação cognitiva em pacientes idosos. In: Netto MP. Gerontologia. São Paulo: Atheneu; 2005. p. 89-174.

12. Price P, Harding K. The Cardiff Wound Impact Schedule: the development of a condition specific questionnaire to assess health-related quality of life in patients with chronic wounds of the lower limb. Int Wound J. 2004;1(1):10-7. https://doi. org/10.1111/j.1742-481x.2004.00007.X.

13. Lino VTS, Pereira SEM, Camacho LAB, Ribeiro Filho ST, Buksman S. Adaptação transcultural da Escala de Independência em Atividades da Vida Diária (Escala de Katz). Cad Saúde Pública. 2008;24(1):103-12. https://doi. org/10.1590/s0102-311X2008000100010. 
14. Augusto FS, Blanes L, Nicodemo D, Ferreira LM. Translation and cross-cultural adaptation of the Cardiff Wound Impact Schedule to Brazilian Portuguese. J Tissue Viability. 2017;26(2):113-8. https://doi.org/10.1016/j.jtv.2016.12.002.

15. Katz S, Akpom CA. A measure of primary sociobiological functions. Int J Health Serv. 1976;6(3):493-508. https://doi. org/10.2190/UURL-2RYU-WRYD-EY3K

16. Bedin LF, Busanello J, Sehnem GD, Silva FM, Poll MA. Estratégias de promoção da autoestima, autonomia e autocuidado das pessoas com feridas crônicas. Rev Gaúcha Enferm. 2014;35(3):61-7. https://doi.org/10.1590/19831447.2014.03.43581.

17. Isaac A, Watson C. How venous leg ulcers affect quality of life. Prim Health Care. 2016;26(3):18-23. https://doi. org/10.7748/phc.26.3.18.530.

18. Kapp S, Santamaria N. The financial and quality-of-life cost to patients living with a chronic wound in the community. Int Wound J. 2017;14(6):1108-20. https://doi.org/10.1111/ iwj.12767.

19. Purcell A, Buckley T, Fethney J, King J, Moyle W, Marshall AP. The effectiveness of EMLA as a primary dressing on painful chroniclegulcers: effectsonwound healingand health-related quality of life. Int J Low Extrem Wounds. 2017;16(3):163-72. https://doi.org/10.1177/1534734617726678.

20. Upton D, Andrews A, Upton P. Venous leg ulcers: What about well-being? J Wound Care. 2014;23(1):14-7. https:// doi.org/10.12968/jowc.2014.23.1.14.

21. Szewczyk MT, Cwajda J, Rogala J, Jawien A. Psychological and social problems of patients with chronic venous disease. Postępy Dermatol Allergol. 2007;24(5):207-10.

22. Molski P, Kruczyński J, Molski A, Molski S. Manual lymphatic drainage improves the quality of life in patients with chronic venous disease: a randomized controlled trial. Arch Med Sci. 2013;20;9(3):452-8. https://doi.org/10.5114/ aoms.2013.35343.
23. Camacho ACLF, Santos RC, Joaquim FL, Louredo DS, Morais IM, Silva EA. Estudo comparativo sobre a capacidade funcional de pacientes adultos e idosos com úlceras venosas. J res fundam care. 2015;7(1):1954-66. https://doi. org/10.9789/2175-5361.2015.v7i1.1.

24. Vieira CPB, Furtado AS, Almeida PCD, Luz MHBA, Pereira AFM. Prevalência e caracterização de feridas crônicas em idosos assistidos na atenção básica. Rev baiana enferm. 2017;31(3):e17397. https://doi.org/10.18471/rbe. v31i3.17397.

25. Rahimi A, Mousavi B, Soroush M, Masumi M, Montazeri A. Pain and health-related quality of life in war veterans with bilateral lower limb amputations. Trauma Mon. 2012;17(2):282-6. https://doi.org/10.5812\%2Ftraumamon.5135.

26. Moreira MMR, Blanes L, Augusto FS, Filho AG, Ferreira LM. Qualidade de vida e capacidade funcional em pacientes com úlcera arterial. Av Enferm. 2016;34(2):170-80. https:// doi.org/10.15446/av.enferm.v34n2.61617.

27. Dias TYAF, Costa IKF, Melo MDM, Torres SMSGSO, Maia EMC, Torres GV. Quality of life assessment of patients with and without venous ulcer. Rev Latino-Am Enfermagem. 2014;22(4):576-81. https://doi.org/10.1590/0104-1169.3304.2454.

28. Allami M, Yavari A, Karimi A, Masoumi M, Soroush M, Faraji E. Health-related quality of life and the ability to perform activities of daily living: a cross-sectional study on 1079 war veterans with ankle-foot disorders. Mil Med Res. 2017;4:37. https://doi.org/10.1186\%2Fs40779-017-0146-1.

29. Abreu AM, Oliveira BGR. A study of the Unna Boot compared with the elastic bandage in venous ulcers: a randomized clinical trial. Rev Latino-Am Enfermagem. 2015;23(4):571-7. https://doi.org/10.1590/0104-1169.0373.2590. 